\title{
Role of MDCT Coronary Angiography with 64-Slice Technology to Detect the Coronary Artery Diseases
}

\author{
Zafar $\mathrm{W}^{1,2 *}$, Farooq $\mathrm{K}^{1}$, Ahmed F1, Rasool F² and Murad $\mathrm{S}^{2}$ \\ ${ }^{1}$ Diagnostic Centre of Radiology and Angiography, Doctors Hospital \& Medical Center, \\ Pakistan \\ ${ }^{2}$ GINUM Cancer Hospital, Pakistan
}

*Corresponding author: Waseem Zafar, Diagnostic Centre of Radiology and

\section{Research Article}

Volume 3 Issue 2

Received Date: March 11, 2019

Published Date: May 23, 2019

DOI: $10.23880 / \mathrm{crij}-16000146$

Angiography, Doctors Hospital \& Medical Center, Pakistan, Tel: 00923004576755; Email: waseem_mt@yahoo.com

\section{Abstract}

Aims: The aim of our study was to investigate the accuracy of 64-slice computed tomography (CT) for assessing hemodynamically significant stenosis of coronary arteries.

Methods and Results: CT angiography was performed in 62 patients (50 male, 12 female) with suspected coronary artery disease and compared with invasive coronary angiography. All vessels were considered for the assessment of significant coronary artery stenosis. Sixty two patients were identified as having significant coronary stenosis on invasive angiography with 56\% (140/248) affected segments. CT correctly identified all 7 patients having no significant stenosis on invasive angiography. Overall sensitivity for classifying stenosis was $96 \%$, specificity was $80 \%$, positive predictive value was $98 \%$, and negative predictive value was $67 \%$.

Conclusion: Sixty-four-slice CT provides a high diagnostic accuracy in assessing coronary artery stenosis.

Keywords: Computed Tomography; Conventional Coronary Angiography; Coronary Artery Disease

Abbreviations: CT: Computed Tomography; MDCT: Multidetector CT; CAD: Coronary Artery Disease; ECG: Electrocardiogram; MSCT: Multi-slice Computer Tomography; QCA: Quantitative Coronary Angiography; MIP: Maximum Intensity projection; MPR: Multiplanar Reconstruction; VRT: Volume Rendering Technique; CABG: Coronary Artery Bypass Graft; CCA: Conventional Coronary Angiography; CTCA: computed Tomography Coronary Angiography; RCA: Right Coronary Artery; LAD: Left Anterior Descending Artery IVUS: Intravascular Ultrasound.

\section{Introduction}

Advances in multidetector CT (MDCT) technology with sub millimeter slice collimation $(0.5 \mathrm{~mm})$ and high temporal resolution ( $(330 \mathrm{~ms})$ permit contrast-enhanced imaging of coronary arteries and coronary plaque during a single breath hold [1]. Appropriate patient preparation, detailed technical and technological knowledge with regard to recognition of typical imaging artifacts (such as beam hardening or motion artifacts), and the adequate choice of post processing techniques to detect stenosis and plaque are prerequisites to achieving diagnostic image quality. A growing number of studies have suggested that 64-slice coronary CT angiography is highly accurate for the exclusion of significant coronary artery stenosis $(>50 \%$ luminal narrowing), with negative predictive values of $97 \%-100 \%$, in comparison with invasive selective coronary angiography. In addition, several studies have indicated that MDCT also can detect calcified and no calcified coronary atherosclerotic plaques 


\section{Clinical Radiology \& Imaging Journal}

[2], especially in proximal vessel segments, showing a good correlation with intracoronary ultrasound. Studies on clinical utility, cost, and cost-effectiveness are now warranted to demonstrate whether and how this technique can change and improve the current management of patients with suspected or confirmed coronary artery disease [3]. Over the last 10 years a dramatic improvement in multi detector CT (MDCT) technology has occurred. The ability to noninvasively image the coronary artery lumen and wall and obtain information on the presence, severity, and characteristics of coronary artery disease (CAD), including the visualization of luminal obstruction and atherosclerotic plaque, constitutes an attractive addition to currently available diagnostic tools, such as nuclear perfusion imaging or invasive selective coronary angiography, for the work-up of patients with known or suspected CAD [3].

\section{Established Modalities in Cardiac Imaging}

Various invasive and non-invasive imaging techniques are in use for cardiac diagnosis. The potential role of CT imaging as a new modality in cardiac disease management derives from the limitations in clinical application and the shortcomings in economic efficacy of the techniques that are available today.

a) Invasive Imaging (Conventional angiography)

b) Noninvasive imaging

- Echocardiography

- Cardiac Perfusion Imaging

- Magnetic resonance Imaging

- CT angiography

\section{Prevalence}

$25 \%$ of all deaths in the USA are related to CAD (Jones and Eaton). About $50 \%$ of these patients die after acute myocardial infarction without prior symptoms. The main risk factors to develop CAD are age, sex (men are at higher risk than women), high blood pressure, high blood lipid level, smoking, diabetes, overweight, family history, lack of exercise and mental stress. Approx. 1 in 226 or $0.44 \%$ or 1.2 million people in USA (source statistic for calculation: estimated 1.2 million new or recurrent coronary attacks in the USA 2004 (American Heart Association).

\section{Incidence Extrapolations for USA for Coronary Heart Disease}

$1,200,000$ per year, 100,000 per month, 23,076 per week, 3,287 per day, 136 per hour, 2 per minute, 0 per second. [Source statistic for calculation: "estimated 1.2 million new or recurrent coronary attacks in the USA 2004 (American Heart Association).

\section{Incidence Extrapolations for USA for Coronary Heart Disease}

$1,200,000$ per year, 100,000 per month, 23,076 per week, 3,287 per day, 136 per hour, 2 per minute, 0 per second. [Source statistic for calculation: "estimated 1.2 million new or recurrent coronary attacks in the USA 2004 (American Heart Association).

\section{Epedimiology}

The main risk factors to develop CAD are Age, Sex (men are at higher risk than women), High blood pressure, High blood lipid level, Smoking, Diabetes, Overweight, Family history, Lack of exercise and mental stress (Assmann et al. 1999). CAD is the symptomatic presentation of coronary atherosclerosis, which causes narrowing of the coronary vessel lumen due to lipid-rich, fibrous or calcified plaques that adhere to the vascular walls. The reduction in blood and oxygen supply causes ischemia in the myocardium. The patient perceives this as an attack of chest pain (angina pectoris) and, in advanced stages, as acute myocardial infarction with necrotic changes of the heart muscle if coronary vessels are severely obstructed or totally occluded.

\section{Instrumentation}

Aquilion 64-Slice MDCT, Toshiba, Japan

$>\quad$ 64-slice scanner has increased slices per gantry rotation (64 slices) and faster gantry speed (330 $\mathrm{ms}$ /rotation), which translate into superior spatial resolution (0.5) and temporal resolution (165 vs. $188 \mathrm{~ms}$ ). The reduction in voxel size makes the distinction between hypo intense soft plaque and blood pool contrast more evident (Figure 1).

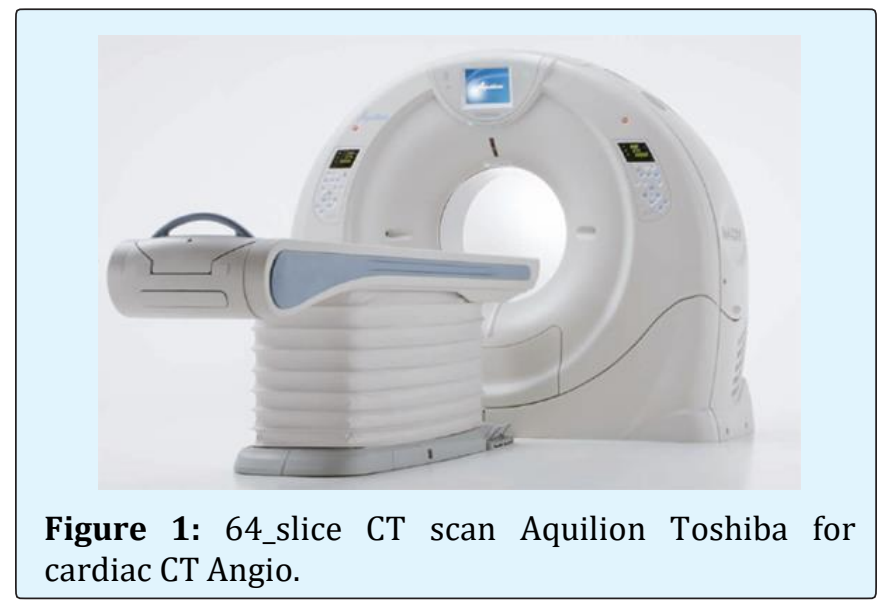




\section{Clinical Radiology \& Imaging Journal}

\section{Objective}

The study is designed with following objectives:

a) To measure the Influence of calcium score \& grading of stenosis on CT Angio and conventional angio.

b) To measure the accuracy of 64-slice MDCT by comparing the Cardiac CTA and Conventional Coronary Angiography.

c) To detect the coronary artery disease through CT Angio \& its comparison with conventional coronary angiography.

\section{Rational}

This study may help:

a) Most of our persons in our community are hypertensive, obese, family History, lack of exercise, Smoker, high Cholesterol level, diabetic etc.

b) Patients and coronary heart disease is main a cause of narrowing or blockage of vessels or causing cardio vascular diseases.

c) CT angiography is initial tool for imaging or screening the cardio vascular diseases (atherosclerosis, stenosis) in those patients.

d) In CT Angiography we can determine the nature of plaque and grading of stenosis can be done. This is noninvasive procedure; fast imaging done in CT angiography and it has high spatial resolution and minimum slice thickness required.

e) Although in angiography (conventional angiography) is gold standard procedure for determining the nature of plaque but for this intra-vascular ultrasound is required which is too much expensive and require arterial puncture (invasive procedure). So CT is tool for initial imaging the location of plaque and determines the nature of plaque and grading of stenosis.

f) Reduce lack of agreement between imaging findings and intraoperative findings.

\section{Material and Methods}

\section{Subject \& Study Design}

This is a non-randomized, observational study design conducted at Heart, Brain \& Body Scan, and Diagnostic Centre of radiology and angiography department of Doctor S' Hospital Lahore.

\section{Study Population}

In 62 consecutive patients scheduled to have invasive coronary angiography for suspected coronary artery disease, MSCT was also performed in same patients. The human investigation committee approved the study protocol, and all patients gave informed consent.

\section{Inclusion Criteria}

a) Suspected coronary artery disease.

b) Differential diagnosis of symptomatic patients with atypical chest pain.

c) Risk stratification in asymptomatic individuals with intermediate to high risk for future myocardial event.

d) Follow up of post stenting or post-CABG patients [4].

\section{Exclusion Criteria}

Exclusion criteria included irregular heart rate, patients at risk for iodinated contrast agents (congestive heart failure, dye allergy, or elevated serum creatinine $>1.5 \mathrm{mg} / \mathrm{dl}$ ), or contraindications to betablocking drugs [4].

\section{Patient Preparation}

Patients not already on beta-blocking drugs received $100 \mathrm{mg}$ of atenolol for heart rates over 65 beats/min, or $50 \mathrm{mg}$ of atenolol for heart rates under 65 beats/min but over 50 beats/min, $1 \mathrm{~h}$ before MSCT imaging. Heart rate, electrocardiogram (ECG), and blood pressure were monitored, and additional intravenous metoprolol (5 to $30 \mathrm{mg}$ ) was administered to achieve a target heart rate $<65$ beats/min [5]. However, no patient was excluded because of a heart rate above this target. Sublingual nitroglycerin $0.4 \mathrm{mg}$ was given $1 \mathrm{~min}$ before image acquisition [3].

\section{Scan Protocol and Image Reconstruction}

All patients were scanned on a 62-slice MSCT scanner (Aquilion 64-Slice MDCT, Toshiba, Japan). An initial nonenhanced ECG-gated scan was performed for calcium scoring [2]. CT scans performed on a 64-slice scanner with a $0.37 \mathrm{~s}$ rotation time A bolus of $80 \mathrm{~mL}$ iodinated contrast media (Visipaque, Ultravist, omnipaque 320 $\mathrm{mg} / \mathrm{mL}$ ) was injected into an antecubital vein at a flow rate of $5 \mathrm{~mL} / \mathrm{s}$, followed by a $50 \mathrm{~mL}$ saline chasing bolus. Start delay was defined by bolus tracking in the ascending aorta and scan start was automatically initiated $5 \mathrm{~s}$ after reaching the threshold [140 HU (HU, Hounsfield Units)]. After this, scanning was performed from the tracheal bifurcation to the diaphragm using the following parameters: X-ray tube potential $120 \mathrm{kV}$, effective tube current $680 \mathrm{~mA}$, slice collimation $64 \times 0.5 \mathrm{~mm}$, table feed $9.2 \mathrm{~mm} /$ rotation, and pitch 0.24 . All CT scans were performed using an implemented fully automated realtime anatomy based dose regulation. The overall scan 


\section{Clinical Radiology \& Imaging Journal}

time was shorter than $12 \mathrm{~s}$ (median $11.2 \mathrm{~s}$, range 10.7$11.9 \mathrm{~s}$ ) and the mean total time for the examination was $<15$ min. We used retrospective electrocardiographic (ECG) gating for optimal heart phase selection [6]. Estimated effective radiation dose was $13 \mathrm{mSv}$ for men and $18 \mathrm{mSv}$ for women. Electrocardiographically gated datasets were reconstructed automatically at $65 \%$ of the R-R cycle length and $35 \%$ of the R-R cycle length to approximate end-systole and end-diastole, respectively. Additional reconstruction windows were constructed after examination of datasets in motion artifacts was present [4] (Figure 2).

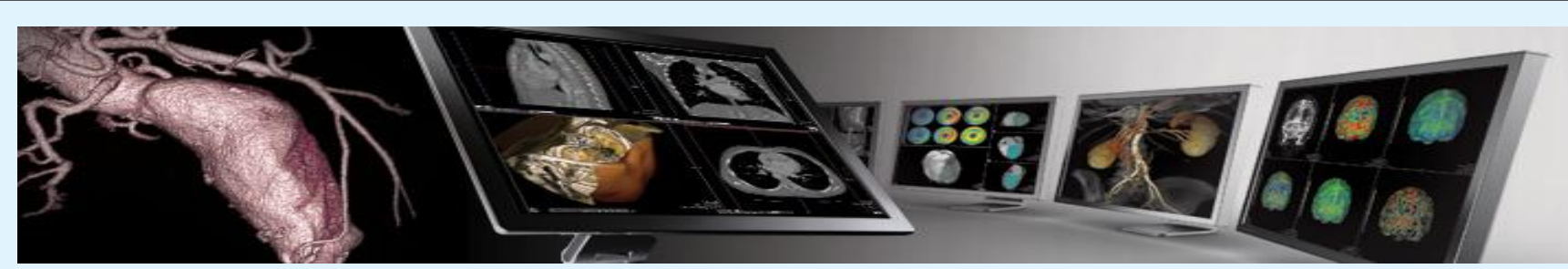

Figure 2: Vitrea 3D post processing workstation for cardiac CT angio.

\section{Statistical Analysis}

\section{MDCT Angiographic Analyses}

Calcium scores in Agatston $U$ were analyzed using CACS software (Aquilion 64-Slice MDCT, Toshiba, Japan); MSCT angiograms were analyzed on a three-dimensional workstation (VITREA). Scans were analyzed by consensus of two observers unaware of the clinical data and blinded to results of the invasive quantitative coronary angiography (QCA). Each lesion identified was examined using MIP (maximum intensity) and MPR (multiplanar reconstruction) VRT, techniques along multiple longitudinal axes and transversely. Lesions were classified by the maximal luminal diameter stenosis seen in any plan. Lesions that could not be quantified to within $25 \%$ diameter stenosis because of hazy or irregular borders were classified by a qualitative severity scale: $0=$ no stenosis, $1=1 \%$ to $25 \%$ stenosis, $2=26 \%$ to $50 \%$, and $3=51 \%$ to $>75 \%$, $=4[4]$.

\section{Invasive Angiographic Analyses}

Invasive coronary angiograms were evaluated by a single observer blinded to the MSCT results. Segmental disease was analyzed in each vessel using the same 15segment model employed for MSCT analysis. Stenosis severity in each segment was classified according to the maximal luminal diameter stenosis present in each segment. Lesions were examined in orthogonal views, and stenosis severity determined.

\section{Comparative Analyses}

Quantitative per-lesion analysis compared the maximal percent diameter stenosis of the most severe lesion in each segment by each imaging modality. The accuracy of MSCT to detect significant disease is compared to invasive QCA according to the following analyses: 1) per-artery analysis, comparing each artery, examining the presence of significant lesions in each of the major coronary vessels (right coronary artery, left circumflex, left anterior descending, and left main) [7]. Per-patient analysis evaluating the presence of any significant lesion in a given patients. In determining MSCT lesion severity, quantitative values were always used if available. For analysis of sensitivity, specificity, and positive and negative predicative accuracy, QCA lesion severity was used as the "gold standard.

\section{Results}

\section{Descriptive Statistical Analysis}

a) Frequency Distribution (Figure 3)

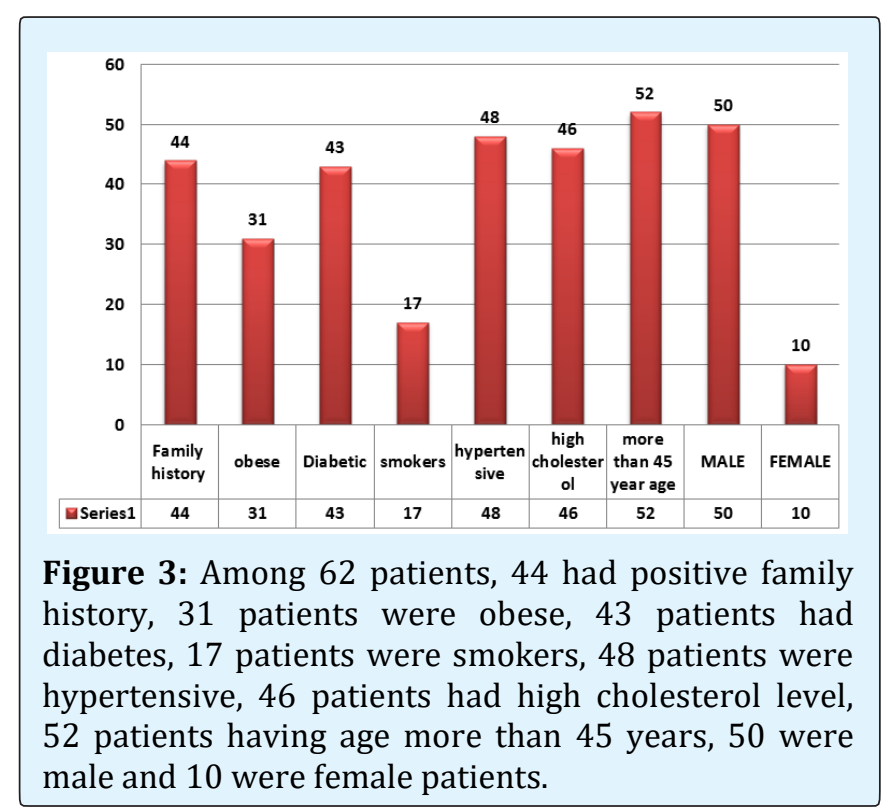




\section{Clinical Radiology \& Imaging Journal}

b)

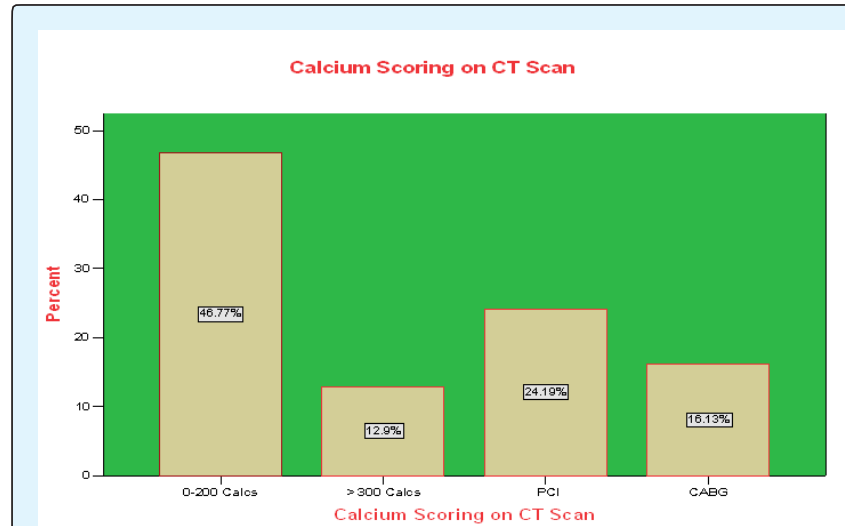

Figure 4: Interpretation: Table 1 \& Figure 4 shows that out of a total of 62 patients, 29 patients (46.8\%) had CACS from $0-100,8$ patients(13\%) have CACS more than 300,15 patients $(24 \%)$ are post -stenting, and 10 patients $(16 \%)$ were post CABG.

\section{Influence of Calcium Score on MSCT Accuracy}

We examined the accuracy of MSCT to detect a significant stenosis in a given patient according to calcium score (Agatston U). The Effect of Coronary Calcification on Diagnostic Accuracy of Coronary MSCT Compared to QCA (Figures 5 \& 6, Table 1).

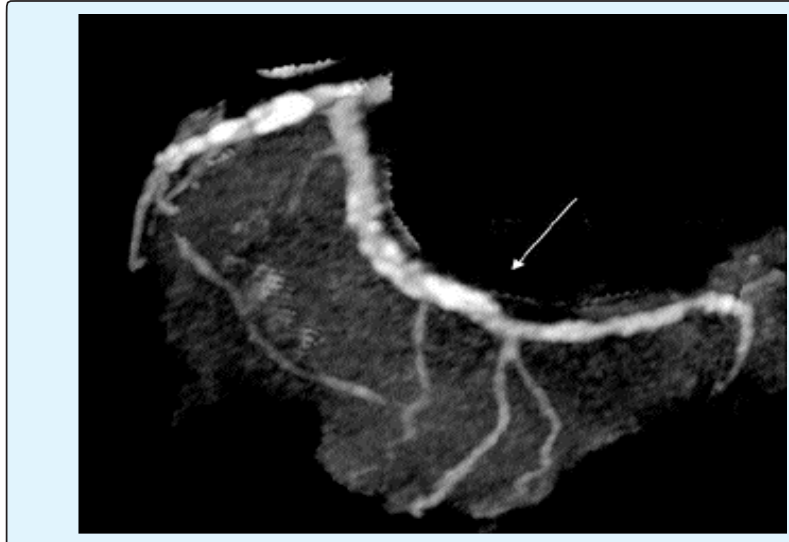

Figure 5: Multiplanar reformat image showing heavily calcified LCX (arrow).

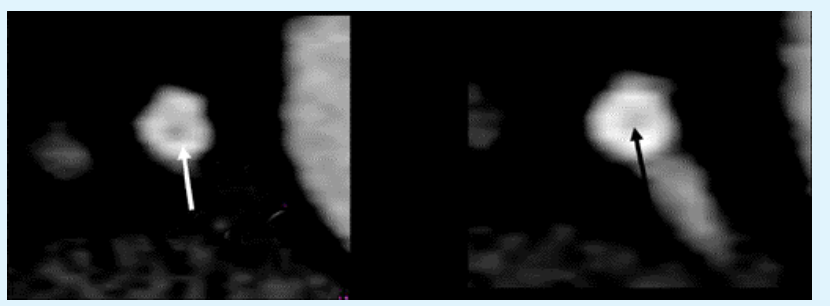

Figure 6: Multiplannar reformat cross-section of the stent lumen showing the low attenuated neointimal hyperplasia causing a moderate stenosis of the stent (arrow). A contiguous section of the same stent distally shows the normal lumen (thick arrow).

\begin{tabular}{|c|c|c|c|c|}
\hline Patients' calcium score $*$ & Sensitivity & Specificity & PPV & NPV \\
\hline $0-100(n=37)$ & $11 / 13(85 \%)$ & $12 / 14(86 \%)$ & $11 / 13(85 \%)$ & $12 / 14(86 \%)$ \\
\hline$>300(n=4)$ & $4 / 4(100 \%)$ & $12 / 14(86 \%)$ & $4 / 6(67 \%)$ & $12 / 12(100 \%)$ \\
\hline
\end{tabular}

Table 1: Showing the accuracy of 64 slice CT for Calcium scoring patients.

\section{Inferential Statistical Analysis}

a) Ho: CTCA = CCA

b) HI: CTCA $=/=$ CCA

c) Level of significance: $95 \%$, Alpha $=0.05$

d) Test Statistics: Spearmen Correlation $=r$

$$
r=\frac{\sum X Y-\frac{\sum X \sum Y}{N}}{\sqrt{\left(\sum X^{2}-\frac{\left(\sum X\right)^{2}}{N}\right)\left(\sum Y^{2}-\frac{\left(\sum Y\right)^{2}}{N}\right)}}
$$

e) MDCT compared to CCA for quantification of lesion severity (Table 2). Calculations:

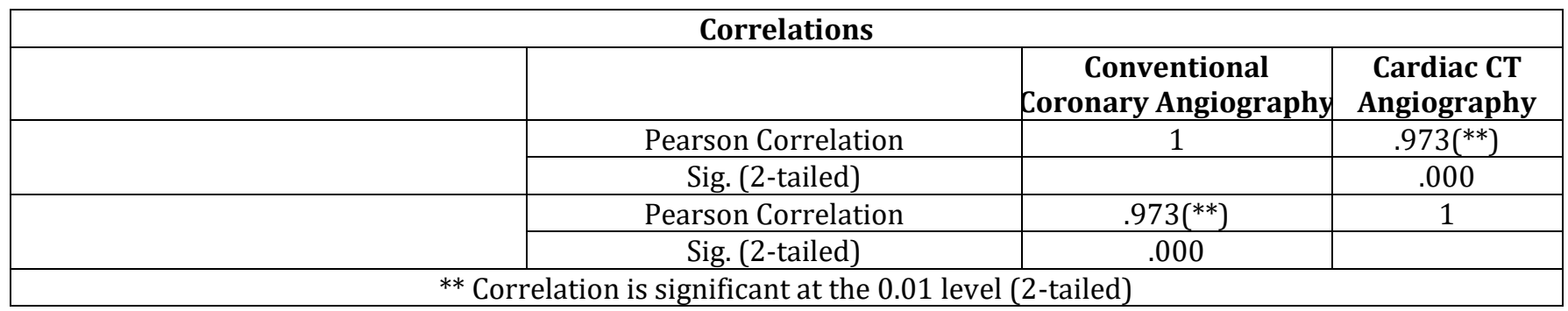

Table 2: The spearman correlation coefficient between the two modalities CTCA and CCA is $0.973(\mathrm{p}<0.05)$. 


\section{Clinical Radiology \& Imaging Journal}

f) Critical region if $\mathrm{P}<\alpha(0.000<0.05)$ then we reject the null hypothesis

g) Since we calculate that spearman correlation and hence we conclude that reject Ho \& accept alternative hypothesis because $\mathrm{P}<\alpha(0.000<0.05) \mathrm{s}$

\section{MSCT Compared to QCA for Detection of Significant Stenosis}

Diagnostic Accuracy of Coronary MSCT Compared to QCA for Detection of Lesions (Table 3)

\begin{tabular}{|c|c|c|c|c|}
\hline & Sensitivity & Specificity & PPV & NPV \\
\hline Patients (n = 62) & $55 / 57(96 \%)$ & $4 / 5(80 \%)$ & $55 / 56(98 \%)$ & $4 / 6(67 \%)$ \\
\hline Arteries (n=248) & $140 / 152(92 \%)$ & $90 / 96(94 \%)$ & $140 / 146(96 \%)$ & $90 / 102(88 \%)$ \\
\hline
\end{tabular}

Table 3: A total of 248 arteries could be analyzed. On a per-artery basis, MSCT had a sensitivity of 92\% (140 of 152) and a specificity of $94 \%$ (90 of 96). The positive predictive value was $96 \%$ (140 of 146), and negative predictive value was $88 \%$ (90 of 102).

All 62 patients could be analyzed for the presence of disease. Multislice CT correctly detected significant disease in 55 of 57 cases for an overall sensitivity perpatient of $96 \%$. Multislice CT documented absence of significant disease in 4 of 5 patients for an overall specificity per-patient of $80 \%$. The positive predictive value for any significant disease was $98 \%$ (55 of 56), and the negative predictive value was $67 \%$ (4 of 6 ). Accurate determination of the presence or absence of significant coronary disease was made in 59 of 62 patients $(95 \%)$ (Figures 7-14).
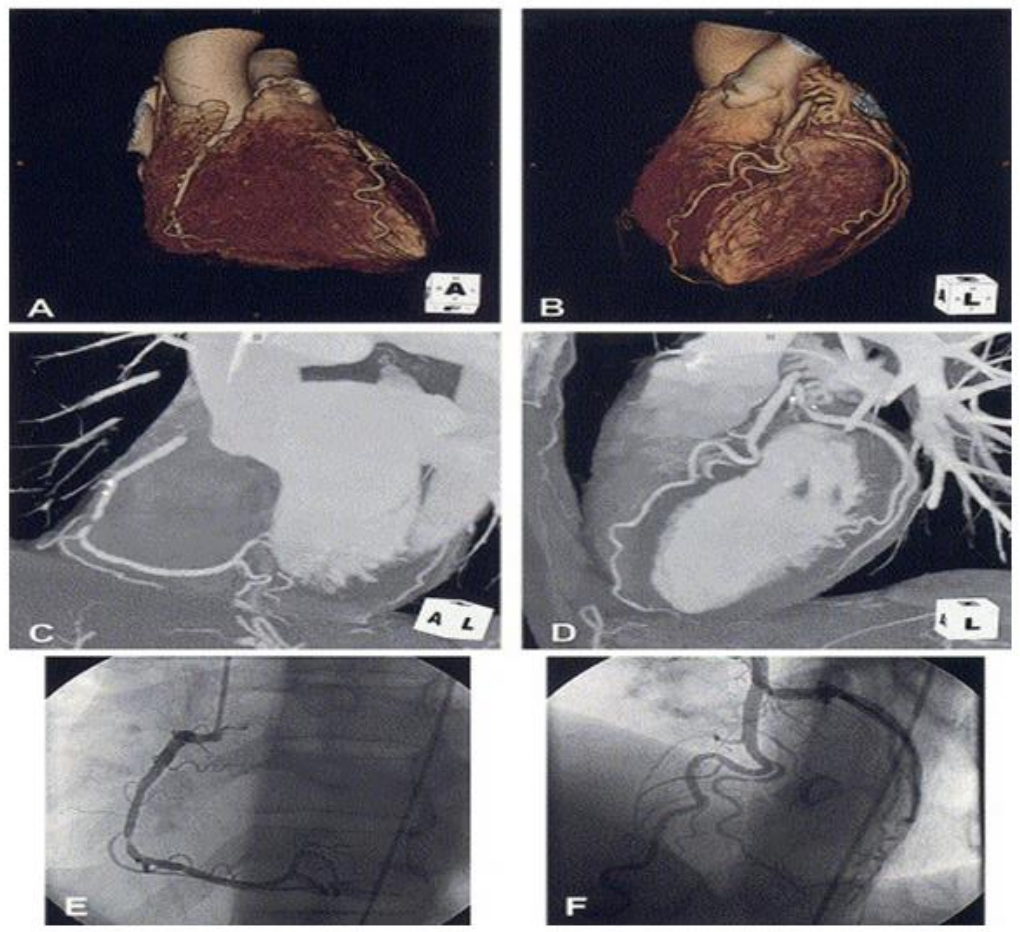

Figure 7: Which showing 3D SSD images \& MIP images of upper two rows \& lower row showing the images of same patient of Angiography RCA \& LAD arteries. 


\section{Clinical Radiology \& Imaging Journal}

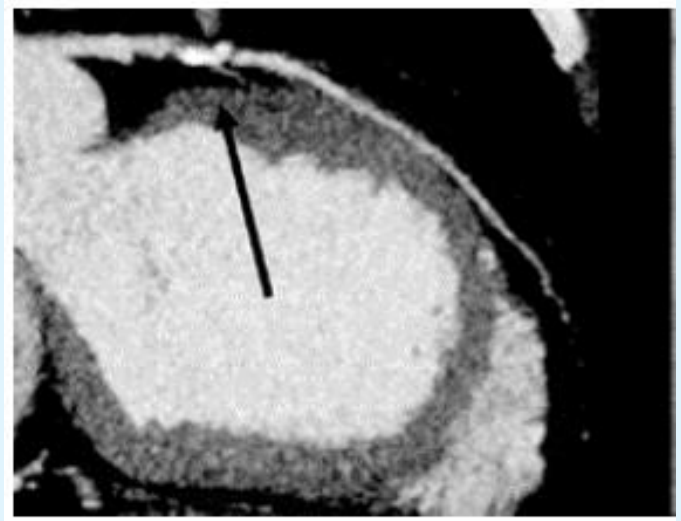

Figure 8: MIP image of LAD showing a high grade stenosis underneath the calcified plaque (arrow).

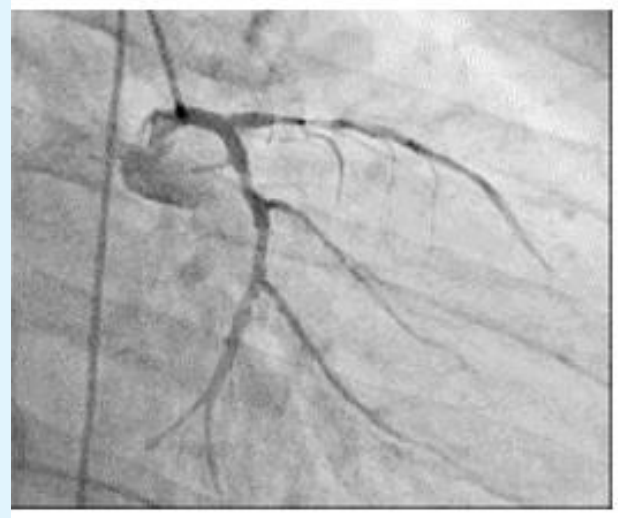

Figure 9: Angiogram showing high grade stenosis of LAD of the same patient.

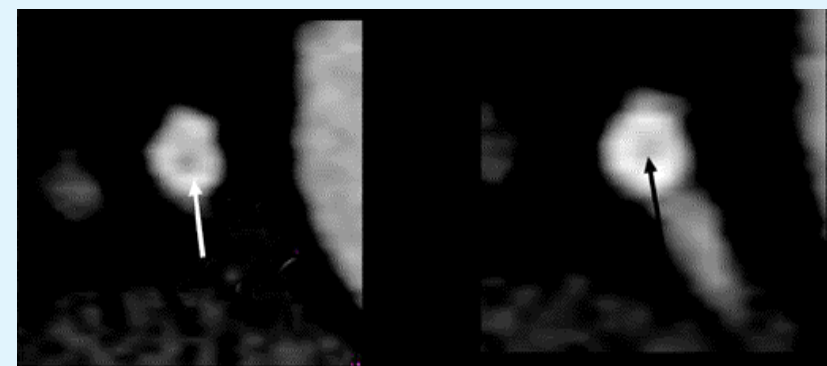

Figure 10: Multiplanar reformat cross-section of the stent lumen showing the low attenuated neointimal hyperplasia causing a moderate stenosis of the stent (arrow). A contiguous section of the same stent distally shows the normal lumen (thick arrow).
Figure 11: Angiogram showing the moderate LAD stent.

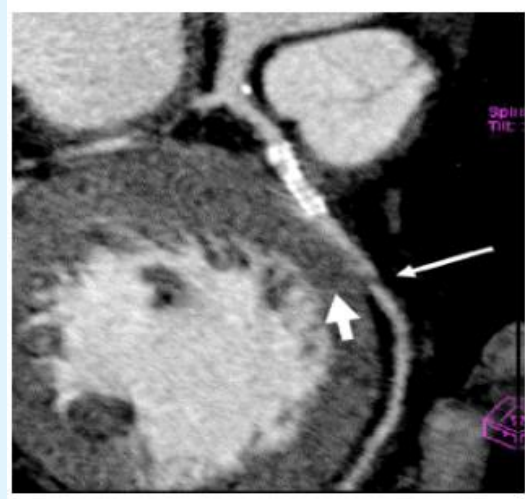

Figure 12: Multiplanar reformat image showing LAD with stent in the proximal part. A step artifact was seen distally mimicking a stenosis segment (arrow). The linear step Artifact was seen across the LV wall (solid arrow) continuing with the step in the LAD Stenosis (arrow) of the same patient.

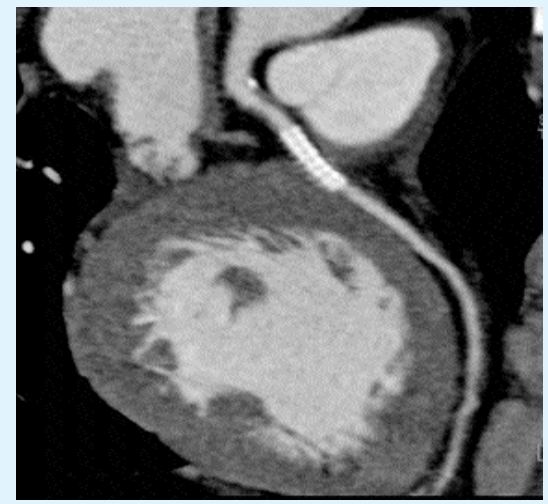

Figure 13: Multiplanar reformat image of the same patient taken in another phase shows normal distal part of the LAD without any step artifact. 


\section{Clinical Radiology \& Imaging Journal}

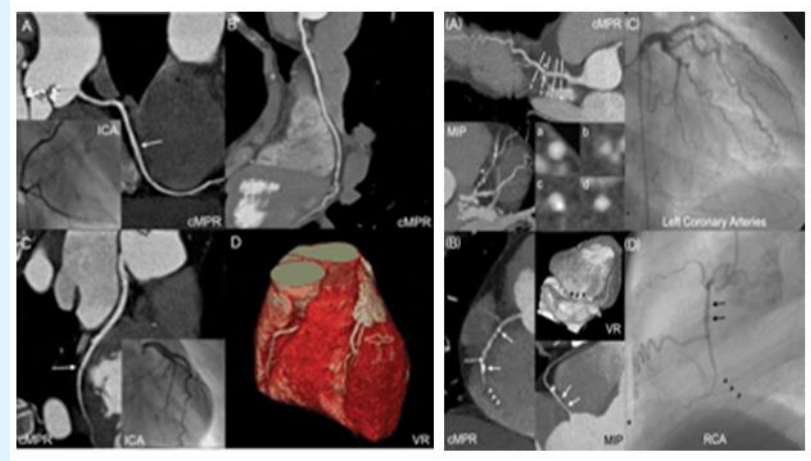

Figure 14: Showing normal images of CABG patients of both modalities CTA \& Angiography.

\section{Discussion}

Observations from this study demonstrate that this 64-slice MSCT scanner consistently provides high-quality noninvasive coronary arteriograms that accurately delineate the presence or absence of significant lesions within the entire coronary tree in a broad spectrum of patients, including those with marked coronary calcification, relatively high heart rates, and obesity. In aggregate, these studies document that noninvasive coronary MSCT imaging can accurately determines the presence or absence of significant coronary lesions. However, prior studies, with one exception employed qualitative analyses only. Furthermore, in contrast to the present study, these prior studies excluded many "realworld" patients because of high heart rates, coronary calcification, or obesity, in addition to excluding all vessels $<1.5 \mathrm{~mm}$ in diameter. In comparison to 16 -slice MSCT scanners, the present 64-slice scanner has increased slices per gantry rotation (64 vs. 16) and faster gantry speed (330 ms/rotation vs. 375$)$, which translate into superior spatial resolution (0.4 vs. $0.5 \mathrm{~mm})$ and temporal resolution (165 vs. $188 \mathrm{~ms}$ ). The reduction in voxel size makes the distinction between hypointense soft plaque and blood pool contrast more evident. Smaller voxel size also reduces partial volume effects, minimizing the degree of calcium blooming and beamhardening artifacts. Partial volume effects are due to averaging of different densities within a single voxel. Calcium deposits are metal density and thus overwhelm the density of other tissues in the same voxel. Beam hardening is due to the attenuation of low-energy X-ray by very dense structures such as calcium. A higher energy beam, causing a darker appearance that can be mistaken for plaque, therefore penetrates adjacent pixels. All these effects can be modified, but not eliminated, by the smaller voxel size produced by the 64-slice scanner. The efficacy of this scanner in ameliorating imaging difficulties is shown in an overall sensitivity of $95 \%$ and specificity of $90 \%$ for the detection of angiographically significant stenoses even in the presence of high coronary calcium scores [1].

\section{Invasive Imaging (Conventional angiography)}

Selective coronary angiography (CA) is considered the gold-standard for diagnosis of the coronary artery lumen [8]. A catheter is inserted into the arterial system via a peripheral artery (Judkins technique) and moved through the aorta into the origins of the coronary arteries. The distribution of contrast agent, administered via the catheter into the coronary arteries, is imaged in several views with planar X-ray technique in order to identify narrowing of the vessel lumen. The maximum 2-dimensional resolution that can be achieved is about 0.15 $\mathrm{mm}$. Basic cardiac function parameters (e.g. ejection fraction) can be derived after administration of contrast agent into the ventricle and assessment of the ventricle size in diastole and systole. For patients with suspected $\mathrm{CAD}$, the diagnostic procedure can be combined with PTCA or stent insertion in the same session. Although evaluation of the vessel wall and of coronary plaques is not possible, CA is currently the standard technique to detect and evaluate coronary artery stenosis and provides a basis for decisions for further work-up. However, due to its invasiveness with possible complications (Harrison) and the considerable amount of applied X-ray dose (3-6 $\mathrm{mSv}$ on average) (Leung and Martin) there is great interest in noninvasive imaging with less $\mathrm{X}$-ray radiation and less direct interaction of the physician.

Evaluation of the vessel wall and of atherosclerotic plaques has become feasible with the advent of intravascular ultrasound (IVUS). IVUS applied to the coronary arteries allows for the assessment of the lumen diameter and plaque dimension. Plaque morphology can 


\section{Clinical Radiology \& Imaging Journal}

be evaluated in terms of lipid content and identification of fibrous and calcified material. However, IVUS has not yet been established as a routine clinical tool because of its high invasiveness, restriction to proximal coronary segments and high costs [6].

\section{Non-invasive Imaging}

The non-invasive cardiac imaging tools that are routinely used today are echocardiography, nuclear imaging and magnetic resonance imaging.

Echocardiography (cardiac ultrasound) allows assessment of shape and thickness of the cardiac walls and of the heart valves. Cardiac function can be evaluated via wall motion, and cardiac blood flow assessment is possible with Doppler sonography techniques. Atherosclerotic disease can be assessed in the carotid arteries and in the great thoracic vessels. However, imaging of the coronary arteries and detection of coronary plaques and stenosis are not yet possible.

Nuclear imaging can be used for evaluation of cardiac function, myocardial perfusion and myocardial viability.

Magnetic resonance imaging MRI): Dedicated scan sequences have been developed for ECG-correlated imaging with high temporal resolution down to 20-50 ms that allow for true 4D imaging of the heart.

\section{Cardiac imaging with MDCT}

a) CT scans performed on a 64-slice scanner with a $0.37 \mathrm{~s}$ rotation time (Aquilion 64-Slice MDCT, Toshiba, Japan). A bolus of $80 \mathrm{~mL}$ iodinated contrast media (Visipaque, Ultravist, omnipaque $370 \mathrm{mg} / \mathrm{mL}$ ) was injected into an antecubital vein at a flow rate of $5 \mathrm{~mL} / \mathrm{s}$, followed by a $50 \mathrm{~mL}$ saline chasing bolus. Start delay was defined by bolus tracking in the ascending aorta and scan start was automatically initiated $5 \mathrm{~s}$ after reaching the threshold [140 HU (HU, Hounsfield Units)]. After this, scanning was performed from the tracheal bifurcation to the diaphragm using the following parameters: X-ray tube potential $120 \mathrm{kV}$, effective tube current $680 \mathrm{~mA}$, slice collimation $64 \times 0.5 \mathrm{~mm}$, table feed 9.2 $\mathrm{mm} /$ rotation, and pitch 0.24. All CT scans were performed using an implemented fully automated realtime anatomy based dose regulation. The overall scan time was shorter than $12 \mathrm{~s}$ (median $11.2 \mathrm{~s}$, range 10.7$11.9 \mathrm{~s}$ ) and the mean total time for the examination was $<15$ min. We used retrospective electrocardiographic (ECG) gating for optimal heart phase selection.

b) The implemented adaptive cardio volume approach was used for data reconstruction. Depending on the heart rate throughout the examination, axial slices were reconstructed synchronized to the ECG by a single sector (65 b.p.m) or two sector algorithm ( $>65$ b.p.m) using data from one or two consecutive heartbeats [2]. When necessary, R-wave indicators were manually repositioned to improve the quality of synchronization. Images were reconstructed at $10 \%$ intervals of the cardiac cycle to allow assessment of coronary arteries at that cardiac phase with minimal vessel motion. Slices with a thickness of $0.75 \mathrm{~mm}$ (increment $0.5 \mathrm{~mm}$ ) and a medium soft-tissue reconstruction kernel were used for evaluating coronary arteries [6].

\section{Conclusion}

My results indicate high quantitative and qualitative diagnostic accuracy of 64-slice MSCT in comparison to QCA in a broad spectrum of patients. The sensitivity for minimal to mild coronary artery diseases is greater of Cardiac CTA as compared to CCA, because MDCT consists of 0.5 collimated slice width and temporal resolution is less than $0.33 \mathrm{sec}$, so spatial resolution is also greater. MDCT has high efficiency to pick very minute calcium plaques in coronary artery vessels. MDCT can also differentiate between soft, fibrous and hard or calcium plaques with the help of different Hounsfield units but CCA cannot differentiate between these plaques.

\section{References}

1. Raff GL, Gallagher MJ, O'Neil WW, Goldstein JA (2005) Diagnostic Accuracy of Non-Invasive Coronary Angiography using 64-slice Spiral Computed Tomography. J American College of Cardiology 46(3): 552-557.

2. Hoffman MHK, Shi H, Schmid FT, Gelman H, Brambs $\mathrm{HJ}$, et al. (2004)Non-invasive Coronary Imaging with MDCT in Comparison to Invasive Conventional Coronary Angiography: A Fast Developing Technology. American J Roentgenology 182(3): 601608.

3. Hoffman U, Ferencik M, Cury RC, Pena AJ (2006) Coronary CT Angiography. J Nucl Med 47(5): 797806.

4. Leschka S, Alkadhi H, Plass A, Desbiolles L, Grünenfelder J, et al. (2005) Accuracy of MSCT coronary angiography with 64-slice technology: first experience. Eur Heart J 26(15): 1482-1487.

5. Ropers D, Baum U, Poehl K, Anders K, Ulzheimer S, et al. (2003) Detection of coronary artery stenoses with 


\section{Clinical Radiology \& Imaging Journal}

thin-slice multi-detector row spiral computed tomography and multiplanar reconstruction. Circulation 107(5): 664-666.

6. Fine JJ, Hopkins CB, Ruff N, Newton FC (2005) 64slice Cardiovascular CTA, a clinical accuracy evaluation. Department of Investigator Initiated Research, South Carolina Heart Centre, Columbia, South Africa.
7. Nieman K, Cademartiri F, Lemos PA, Raaijmakers R, Pattynama PMT, et al. (2002) Reliable noninvasive coronary angiography with fast submillimeter multislice spiral computed tomography. Circulation 106(16): 2051-2054.

8. Bedi HS, Accuracy of 64-slice CT Coronary Angiography. Christian Medical College and Hospital, Ludhiania, India. 